\title{
LETTER
}

Chronic lymphocytic leukemia

\section{TCL1 transgenic mice as a model for CD49d-high chronic lymphocytic leukemia}

\author{
Eva Szenes ${ }^{1}$ - Andrea Härzschel ${ }^{1,2} \cdot$ Sarah Decker $^{2} \cdot$ Erika Tissino $^{3}$ - Justine Pischeli ${ }^{1}$. Julia Christine Gutjahr ${ }^{1}{ }^{1}$. \\ Sandra Kissel $\left.\right|^{2}$ - Sandra Pennisi ${ }^{2}$ - Jan Philip Höpner ${ }^{1}$ - Alexander Egle $\mathbb{D}^{1}{ }^{1}$. Nadja Zaborsky ${ }^{1}$ - Christine Dierks ${ }^{2}$.

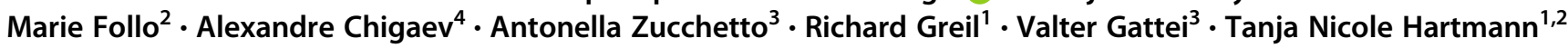

Received: 16 September 2019 / Revised: 20 January 2020 / Accepted: 11 February 2020

(c) The Author(s) 2020. This article is published with open access

\section{To the Editor:}

With the advent of small molecule inhibitors, sustained responses in chronic lymphocytic leukemia (CLL) patients have been achieved. Ibrutinib, an inhibitor of Bruton's tyrosine kinase (BTK), is a particularly potent treatment for CLL, with overall response rates of $82 \%$ in treatment-naive and $43 \%$ in relapsed-refractory patients [1]. These responses are accompanied by transient lymphocytosis in $50-70 \%$ of patients and a concomitant reduction of tumor burden in lymphoid organs [2]. We have recently demonstrated that the high-risk, CD49d-high CLL subgroup [3] displays reduced lymphocytosis and minor lymph node shrinkage upon ibrutinib treatment [4]. CD49d is the alpha subunit of the integrin VLA-4, which plays an essential role in the tissue retention of cells, orchestrating their adhesion to stromal cells, e.g., follicular dendritic cells (fDCs), which in turn express the VLA-4 ligand VCAM-1. To facilitate this strong adhesion,

These authors contributed equally: Eva Szenes, Andrea Härzschel

Supplementary information The online version of this article (https:// doi.org/10.1038/s41375-020-0759-3) contains supplementary material, which is available to authorized users.

Tanja Nicole Hartmann

tanjanhartmann@gmail.com

1 Department of Internal Medicine III with Hematology, Medical Oncology, Hemostaseology, Infectiology and Rheumatology, Oncologic Center, Salzburg Cancer Research InstituteLaboratory for Immunological and Molecular Cancer Research (SCRI-LIMCR), Paracelsus Medical University, Salzburg, Austria

2 Department of Internal Medicine I, Medical Center and Faculty of Medicine, University of Freiburg, Freiburg, Germany

3 Clinical and Experimental Onco-Hematology Unit, Centro di Riferimento Oncologico di Aviano (CRO) IRCCS, Aviano, Italy

4 Department of Pathology and Cancer Center, University of New Mexico, Albuquerque, NM, USA
VLA-4 needs to be activated [5]. We have demonstrated that in CLL the extended, high-affinity conformation of VLA-4 can be triggered by $\mathrm{B}$ cell receptor (BCR) stimulation [4]. Notably, upon treatment with ibrutinib, CD49d-high CLL cells maintain residual BCR-mediated inside-out activation of VLA-4, thus explaining the reduced clinical response of CD49d-expressing CLL [4].

E $\mu$-TCL1-transgenic (TCL1-tg) mice represent a widely used mouse model for CLL [6]. By using this model, here we found that: (i) murine leukemic cells express high levels of surface CD49d; (ii) the BCR-VLA-4 axis in TCL1-tg mice, as investigated upon BCR stimulation and/or exposure to BCR inhibitors, resembles that of the human CD49d-high CLL cohort; (iii) the in vivo distribution of TCL1-tg leukemic cells is dependent on VLA-4-mediated interactions with the microenvironment. Our data demonstrate the value of TCL1-tg mice as a model for CD49dhigh CLL and corroborate the importance of retained BCRinduced VLA-4 activation in a clinical setting.

First, to compare VLA-4 expression patterns between human CLL cells and TCL1-tg cells, we measured CD49d expression of human or murine healthy B cells and leukemic cells by flow cytometry (antibodies: see Supplementary Table 1). Human blood samples $(N=406$; Supplementary Table 2) were obtained upon written informed consent and ethical approval. Blood-derived B cells from age-matched healthy individuals $(N=32)$ and CD49d-high CLL cells $(N=116)$ expressed similar levels of CD49d (Fig. 1ai). Previous reports have shown that CD49d-high CLL cells express lower CD49d levels than healthy B cells. However, our healthy donor group consisted of strictly age-matched individuals (average of healthy cohort: 65.8; CLL cohort: 69.63 years), indicating that CD49d expression might be reduced by age in humans. Moreover, CD49d expression of blood-derived B cells from age-matched C57BL/6J wild-type (WT) mice was similar to that of leukemic cells from TCL1$\operatorname{tg}$ mice (Fig. 1aii). To investigate whether the expression of 
Ai

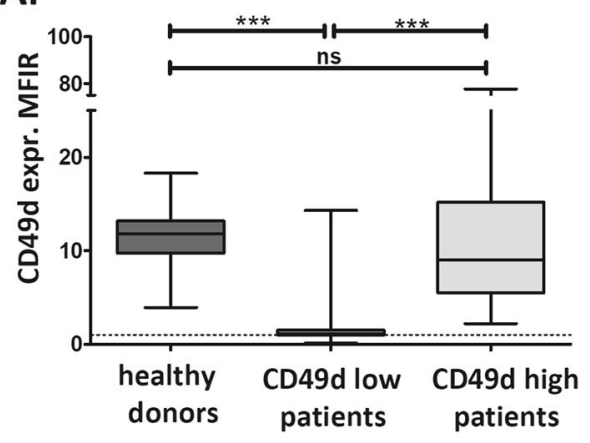

$N=$

$\mathrm{Bi}$

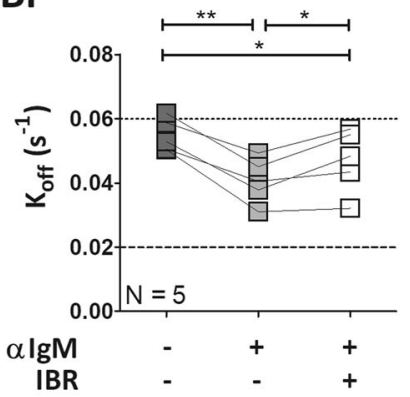

Bii

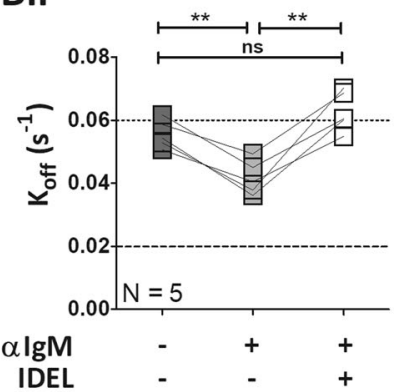

Aii

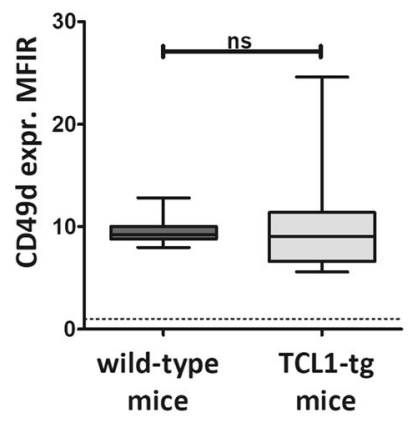

$N=12$

12
Biii

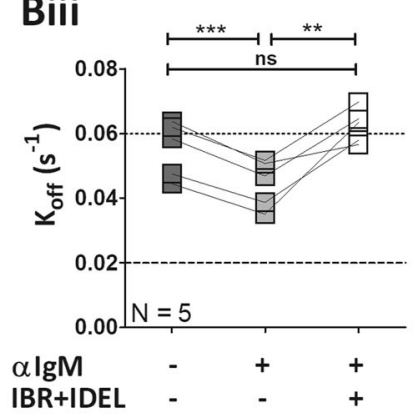

Ci

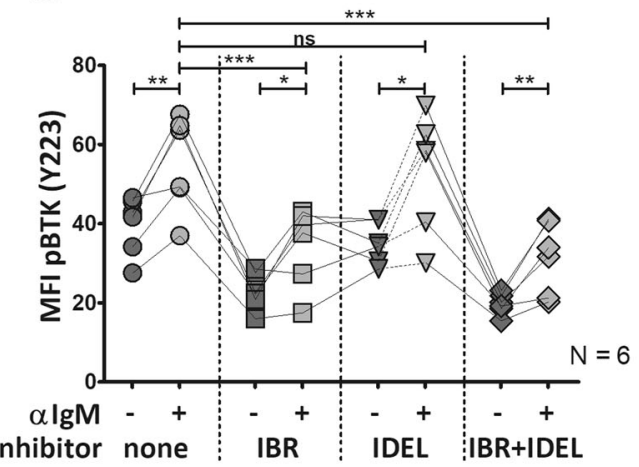

\section{Ciii}

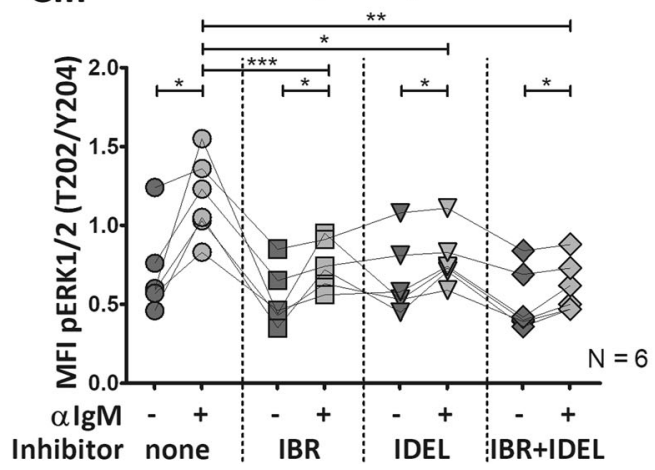

the VLA-4 subunits (CD49d and CD29) had an organspecific pattern, we measured B cells and leukemic cells of spleen (SPL), bone marrow (BM), lymph nodes (LN), blood (BL), liver (LIV), and the peritoneal cavity (PC) from WT
Cii
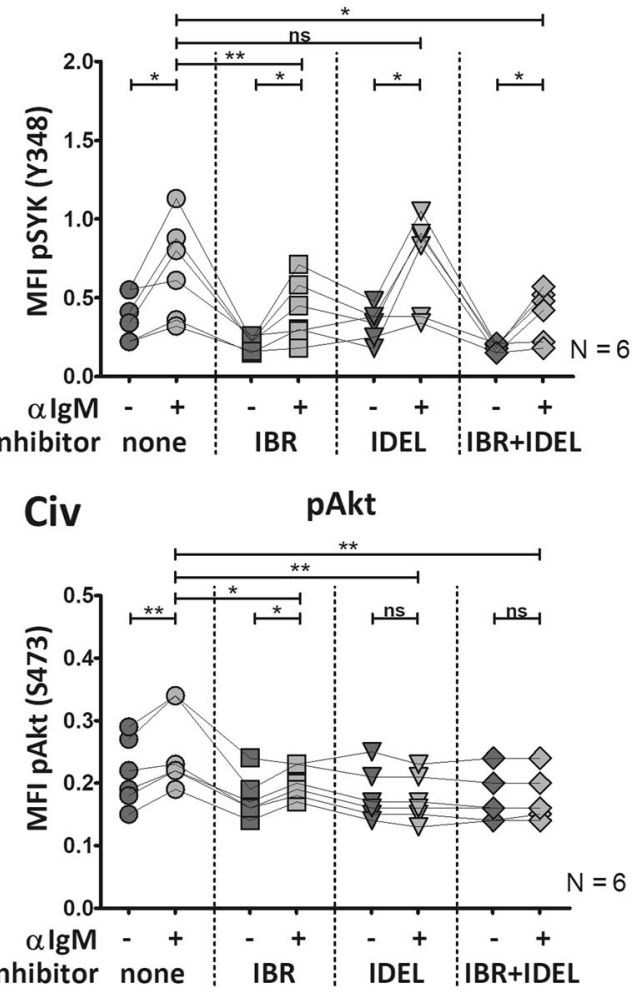

and TCL1-tg mice. Remarkably, expression levels of both integrin subunits were similar in all organs. CD49d mean fluorescence intensity ratio (MFIR) was comparable in WT and TCL1-tg mice (mean MFIR of $\mathrm{WT}=9.893$; 
4 Fig. 1 TCL1-tg leukemic cells resemble human CD49d-high CLL cells in their CD49d expression and VLA-4 inside-out signaling. a The expression of the rate-limiting alpha subunit of VLA-4, CD49d, was measured by flow cytometry. Samples collected from peripheral blood of age-matched (Ai) healthy donors $(N=32)$, CD49d-low $(N=$ 290) or CD49d-high CLL patients $(N=116)$, and (Aii) C57BL/6 J wild-type $(N=12)$ or TCL1-tg mice $(N=12)$ were stained with antiCD5 and anti-CD19, as well as CD49d-specific or its isotype control antibody. MFIR mean fluorescence intensity ratio (human measurements: arithmetic mean, mouse measurements: geometric mean). Dotted line: $\mathrm{MFIR}=1$. Unpaired $t$ tests were performed by GraphPad Prism5. b Purified murine CD5+/CD19+ cells $(N=5)$ were treated for $1 \mathrm{~h}$ with/without $1 \mu \mathrm{M}$ (Bi) ibrutinib (IBR), (Bii) idelalisib (IDEL), or (Biii) both. The dissociation rate $\left(k_{\text {off }}\right)$ of VLA- 4 and its ligand LDV was determined by real-time flow cytometry. $K_{\text {off }}$ values are shown upon different stimulation states: resting (negative control), anti-IgMstimulated $\left(\mathrm{F}\left(\mathrm{ab}^{\prime}\right)_{2}, 10 \mu \mathrm{g} / \mathrm{ml}\right)$, and pretreated + anti-IgM-stimulated. A $k_{\text {off }}$ value above $0.06 \mathrm{~s}^{-1}$ (dotted line) is considered "low affinity", while values below $0.02 \mathrm{~s}^{-1}$ (dashed line) are "high affinity." Connecting lines indicate values from the same tumor. Nonlinear fit "Plateau followed by one-phase decay" and paired $t$ tests were performed using GraphPad Prism5. c Ibrutinib and idelalisib differentially affect BCR signaling in TCL1-tg mice. Splenocytes from TCL1-tg mice $(N=6)$ were pretreated with/without ibrutinib, idealisib or both $(1 \mu \mathrm{M}, 1 \mathrm{~h})$. Cells were stained with anti-CD3 for $\mathrm{T}$ cell exclusion and with fixable viability dye, followed by a stimulation by anti-IgM $\left(\mathrm{F}\left(\mathrm{ab}^{\prime}\right)_{2}, 10 \mu \mathrm{g} / \mathrm{ml}, 5 \mathrm{~min}\right)$. Cells were fixed and permeabilized, stained with phosflow antibodies, and measured by flow cytometry. The mean fluorescence intensity (MFI, geometric mean) values of (Ci) pBTK, (Cii) pSYK, (Ciii) pERK1/2, and (Civ) pAkt of viable CD3-negative cells are shown. To validate whether the CD3-negative cell population corresponds with the leukemic cells, the samples were stained with anti-CD5 and anti-CD19 in parallel. Connecting lines indicate values from the same tumor. Paired $t$ tests were performed by GraphPad Prism5. $* P<0.05 ; * * P<0.01 ; * * * P<0.001$, ns nonsignificant.

TCL1-tg =9.710) (Supplementary Fig. 1a), while the expression of the CD49d-coupled beta subunit CD29 (beta1) was higher in TCL1-tg mice (mean MFIR of WT $=7.261$; TCL1-tg $=21.920$ ) (Supplementary Fig. 1b). Altogether, our data demonstrate that the TCL1-tg model can be used as a murine surrogate for the CD49d-high CLL cohort, with stable expression. As expression levels in the spleen and blood were comparable, and their cellular compositions are highly similar, we used murine splenocytes in subsequent experiments.

To relate the CD49d expression pattern to function, we investigated the mechanistic basis of VLA- 4 activation by BCR stimuli. In human CLL, BCR-induced VLA-4 activation can be detected by the conformation-sensitive anti-human CD29 antibody HUTS-21 [4, 7], which does not recognize the murine epitope. Therefore, here we used the small molecule VLA-4 ligand LDV [8] and measured its binding to leukemic cells by flow cytometry as outlined in Supplementary Fig. 2. With this experimental layout, we obtained real-time information about the kinetics of VLA-4 affinity regulation resulting in enhanced ligand binding. The speed by which unlabeled LDV replaces its FITCconjugated counterpart $\left(k_{\text {off }}\right)$ is used to determine integrin affinity [8]. If VLA-4 and its ligand bind with high affinity, dissociation of LDV-FITC is slower and the replacement time is longer, resulting in lower $k_{\text {off }}$ values (below $0.02 \mathrm{~s}^{-1}$ ). Conversely, low VLA-4-ligand affinity leads to fast LDV-FITC dissociation, shorter replacement time, and higher $k_{\text {off }}$ values (above $0.06 \mathrm{~s}^{-1}$ ). When purified TCL1-tg leukemic cells were stimulated with anti-mouseIgM, VLA-4 affinity increased, with a decrease in $k_{\text {off }}$ from 0.055 to $0.04 \mathrm{~s}^{-1}$. This shows for the first time that in TCL1-tg mice BCR stimulation induces changes in VLA-4 conformation. Ibrutinib pretreatment did not fully prevent this inside-out activation of VLA-4 (Fig. 1bi), recapitulating the observations in human CD49d-high CLL cells [4]. Next, we treated the cells with the phosphoinositide-3-kinase delta (PI3Kס) inhibitor idelalisib [9]. Treatment of leukemic cells with idelalisib (Fig. 1bii) and the ibrutinib/idelalisib combination (Fig. 1biii) was highly effective with no difference between the affinities of unstimulated compared with stimulated + idelalisib-treated or stimulated + combinationtreated cells. In human CLL, neither ibrutinib nor idelalisib is sufficient on its own to prevent inside-out signaling via the BCR [4], suggesting that the stimulation can bypass both BTK and PI3Kס. In TCL1-tg mice, PI3K $\delta$ seems to be a dominant molecule that mediates inside-out signaling between the BCR and VLA-4.

To test that the residual BCR-induced VLA-4 activation was not a result of insufficient inhibition by ibrutinib, we measured the phosphorylation of signaling molecules of the BCR pathway in TCL1-tg splenocytes treated with/without BCR inhibitors by phosflow. Ibrutinib and idelalisib showed specific and expected patterns of inhibition. Singleagent ibrutinib treatment significantly reduced the phosphorylation of BTK (Fig. 1ci) and spleen tyrosine kinase (SYK, Fig. 1cii) after BCR stimulation. Extracellular signalregulated kinase 1/2 (ERK1/2, Fig. 1ciii) phospho signal was decreased by both inhibitors. The phosphorylation of Akt (Fig. 1civ), the downstream signaling component of PI3K, was efficiently inhibited by idelalisib. Both inhibitors showed expected inhibition, therefore, the residual BCRmediated VLA-4 activation in ibrutinib-treated cells was not a result of suboptimal BTK blocking.

Having examined the connection between the BCR and VLA-4 in vitro, we next investigated the importance of VLA-4 in engraftment and early disease onset of TCL1-tgdriven murine leukemia in vivo. Therefore, we transplanted WT mice with primary TCL1-tg splenocytes [10] and treated the recipients with vehicle or the small molecule CD49d-inhibitor firategrast [11]. Recipient mice were sacrificed and their organs analyzed by flow cytometry 21 days post transplantation. Firategrast-treated mice showed an overall reduction of leukemic cells in the spleen (Fig. 2ai), accompanied by significant spleen weight reduction (Fig. 2aii). Since leukemic cells need to enter the follicles in the white pulp of the spleen and access fDCs 

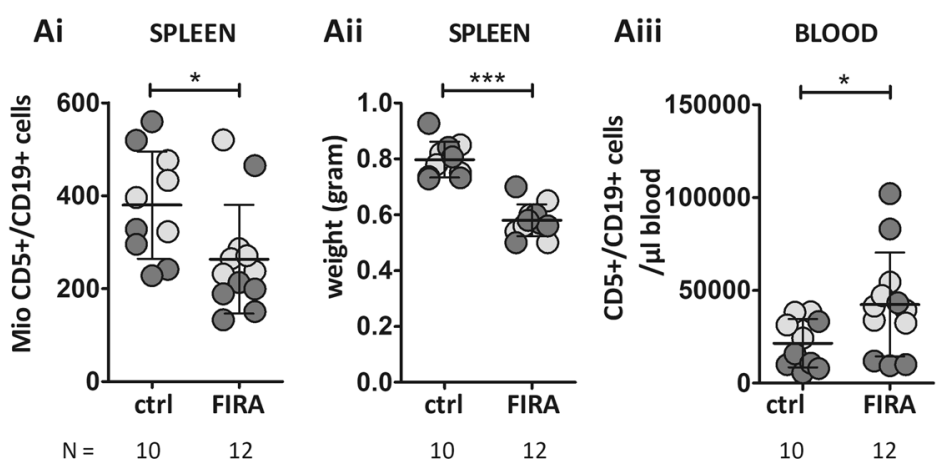

Aiv BONE MARROW
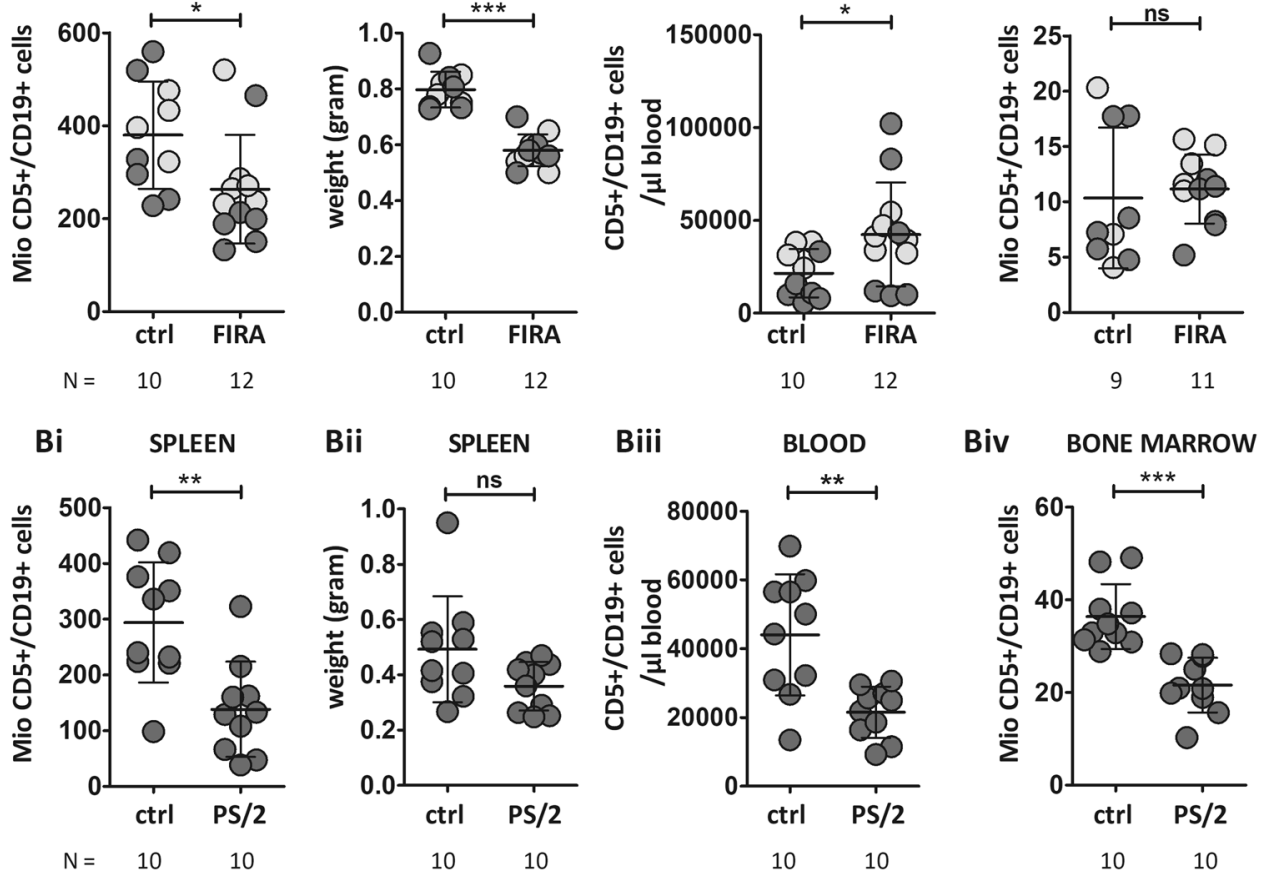

Fig. 2 Inhibition of CD49d in mice transplanted with TCL1-tg splenocytes prevents the engraftment of leukemic cells short-term in the spleen and long-term in the spleen and bone marrow. a Wild-type C57BL/6J mice were transplanted intravenously with $\sim 5$ Mio splenocytes from a TCL1-tg mouse, then treated with vehicle $(0.9 \%$ $\mathrm{NaCl}, 1 \% \mathrm{HP}-\beta-\mathrm{CD})(N=10$, bone marrow $N=9)$ or the small molecule inhibitor firategrast $(N=12), 30 \mathrm{mg} / \mathrm{kg} /$ day in drinking water, starting two (light gray) or seven (dark gray) days post transplantation. Tumor development was monitored by measuring CD5 and CD19 from blood samples from the tail vein twice a week. Mice were sacrificed 21 days after transplantation. Organ infiltration and tumor burden were measured by cell counting (EVE automatic counter) and flow cytometry, with CD5/CD19 staining identifying leukemic cells. b Wild-type

expressing VCAM-1 and providing proliferative signals [12], VLA-4 inhibition prevents the cells from staying in this supportive niche. Indeed, we found a significant increase of CD5+/CD19+ cells in the blood (Fig. 2aiii) upon VLA-4 inhibition, without changes in tumor infiltration in the BM (Fig. 2aiv). This finding was surprising given the prominent role of VLA-4 in CLL retention in the BM [13], but at this stage of engraftment the amount of leukemic cells was still low, and TCL1-tg tumor growth is driven by the spleen, especially in early stages [14].

To investigate a more advanced disease stage, we repeated the treatment study using the inhibitory rat-anti-mouse CD49d antibody PS/2 (Fig. 2b, treatment schedule: see legend). For long-term treatment, we switched to antibody therapy because firategrast has a fast turnover in the organism, although the in vitro inhibitory effects on VLA-4 ligand binding of the two substances were comparable (confirmed by adhesion assays, data not shown). The mice were sacrificed 6 weeks post transplantation. Analysis of the organs

C57BL/6J mice were transplanted intraperitoneally with 25 Mio CLL cells from a TCL1-tg mouse, then treated with the CD49d-blocking antibody PS $/ 2(N=10), 10 \mathrm{mg} / \mathrm{kg}$, or isotype control $(N=10)$, injected intraperitoneally twice a week, starting 7 days post transplantation. Mice were sacrificed 5 weeks after treatment start ( 6 weeks post transplantation). Tumor burden was determined by CD5/CD19 staining and manual cell counting. The absolute numbers of malignant cells from the (Ai, Bi) whole spleen, along with its (Aii, Bii) weight, from (Aiii, Biii) blood (normalized to $\mu \mathrm{l}$ ) and from the (Aiv, Biv) bone marrow (extrapolated from the femora to the whole bone marrow as described in Supplementary Methods) were determined. Unpaired $t$ tests were performed by GraphPad Prism5. Error bars represent standard deviation (SD). $* P<0.05 ; * * P<0.01 ; * * * P<0.001$, ns nonsignificant.

revealed decreased tumor burden in the spleen (Fig. 2bi), along with slightly decreased spleen weight (Fig. 2bii). At this stage, a significant decrease of the tumor load in the blood of PS/2-treated mice was also observed (Fig. 2biii); furthermore, tumor burden in the BM of PS/2-treated mice was significantly decreased (Fig. 2biv). Further investigations are needed to determine whether the redistribution by CD49d inhibition might overcome drug resistance or improve the effectiveness of other therapeutic agents by detaching CLL cells from VCAM-1-expressing stromal cells.

Based on our findings, we propose that TCL1-tg mice represent an adequate model for CD49d-high CLL patients, and provide evidence that BCR-induced VLA-4 inside-out activation remains functional upon treatment with ibrutinib, being in turn hampered upon exposure to idelalisib. This indicates that PI3K, rather than BTK, is an essential part of the signaling between the BCR and VLA-4 in the TCL1-tg model, in part confirming the observation made in humans [4]. Furthermore, we specified the role of CD49d in disease 
development by directly targeting this integrin in vivo, which led to a redistribution of TCL1-tg cells transplanted into WT mice. From a clinical perspective, we suggest that VLA-4 inhibition or additional targeting of BCR pathway molecules, e.g., by PI3K inhibitors other than idelalisib to avoid its severe side effects [15] and potentially targeting different isoforms, can be promising additions to ibrutinib therapy for high-risk CD49d-high CLL patients. This could help to overcome residual BCR-mediated signaling bypassing BTK and mediating tissue retention facilitated by VLA- 4 .

Acknowledgements We thank all patients for their participation in this study. This work was supported by the PhD program Immunity in Cancer and Allergy (W1213, Austrian Science Fund, FWF) to TNH and RG; the Austrian Science Fund (FWF) P26421, the Paracelsus Medical University Salzburg Project E-17-25-130-HAH and the Forschungskomission Freiburg to $\mathrm{TNH}$; the Associazione Italiana Ricerca Cancro (AIRC), Investigator Grant IG-21687 and the Progetto Ricerca Finalizzata PE 2016-02362756, Ministero della Salute, Rome, Italy to VG; and the Austrian Science Fund projects P26719B19, I1299-B21 and I3282-B26 (FOR 2036) and I2795-B28 (ERANET TRANSCAN-2 program JTC 2014-project FIRE-CLL) to AE. We thank Alexandra Hödlmoser and Ursula Denk for mouse handling support.

\section{Compliance with ethical standards}

Conflict of interest The authors declare that they have no conflict of interest.

Ethical approval Human blood samples were obtained upon written informed consent (Ethics Committee Salzburg approval: 415E/1287/4-2011, 415-E/1287/13-2016) from CLL patients at the Department of Internal Medicine III, Paracelsus Medical University, Salzburg, Austria. All mouse experiments were performed under the approval of the Austrian or German Animal Ethics Committee (BMWF-66.012/0030-II/3b/2013, BMWF-66.012/0009-II/3b/2012, 20901-TVG/52/11-2012, 20901-TVG/89/7-2014, BMBWF66.012/0002-V/3b/2018, G18/155).

Publisher's note Springer Nature remains neutral with regard to jurisdictional claims in published maps and institutional affiliations.

Open Access This article is licensed under a Creative Commons Attribution 4.0 International License, which permits use, sharing, adaptation, distribution and reproduction in any medium or format, as long as you give appropriate credit to the original author(s) and the source, provide a link to the Creative Commons licence, and indicate if changes were made. The images or other third party material in this article are included in the article's Creative Commons licence, unless indicated otherwise in a credit line to the material. If material is not included in the article's Creative Commons licence and your intended use is not permitted by statutory regulation or exceeds the permitted use, you will need to obtain permission directly from the copyright holder. To view a copy of this licence, visit http://creativecommons. org/licenses/by/4.0/.

\section{References}

1. Deeks ED. Ibrutinib: a review in chronic lymphocytic leukaemia. Drugs. 2017;77:225-36.

2. Barrientos JC, Burger JA, Byrd JC, Hillmen P, Zhou C, Ninomoto $\mathrm{J}$, et al. Characterizing the kinetics of lymphocytosis in patients with chronic lymphocytic leukemia treated with single-agent ibrutinib. Leuk Lymphoma. 2019;60:1000-5.

3. Bulian P, Shanafelt TD, Fegan C, Zucchetto A, Cro L, Nuckel H, et al. CD49d is the strongest flow cytometry-based predictor of overall survival in chronic lymphocytic leukemia. J Clin Oncol. 2014;32:897-904.

4. Tissino E, Benedetti D, Herman SEM, Ten Hacken E, Ahn IE, Chaffee KG, et al. Functional and clinical relevance of VLA-4 (CD49d/CD29) in ibrutinib-treated chronic lymphocytic leukemia. J Exp Med. 2018;215:681-97.

5. Arana E, Harwood NE, Batista FD. Regulation of integrin activation through the B-cell receptor. J Cell Sci. 2008;121:2279-86.

6. Bichi R, Shinton SA, Martin ES, Koval A, Calin GA, Cesari R, et al. Human chronic lymphocytic leukemia modeled in mouse by targeted TCL1 expression. Proc Natl Acad Sci USA. 2002;99:6955-60.

7. Chigaev A, Waller A, Amit O, Halip L, Bologa CG, Sklar LA. Real-time analysis of conformation-sensitive antibody binding provides new insights into integrin conformational regulation. $\mathrm{J}$ Biol Chem. 2009;284:14337-46.

8. Chigaev A, Blenc AM, Braaten JV, Kumaraswamy N, Kepley CL, Andrews RP, et al. Real time analysis of the affinity regulation of alpha 4-integrin. The physiologically activated receptor is intermediate in affinity between resting and $\mathrm{Mn}(2+)$ or antibody activation. J Biol Chem. 2001;276:48670-8.

9. O’Brien SM, Lamanna N, Kipps TJ, Flinn I, Zelenetz AD, Burger $\mathrm{JA}$, et al. A phase 2 study of idelalisib plus rituximab in treatmentnaive older patients with chronic lymphocytic leukemia. Blood. 2015;126:2686-94.

10. Hofbauer JP, Heyder C, Denk U, Kocher T, Holler C, Trapin D, et al. Development of CLL in the TCL1 transgenic mouse model is associated with severe skewing of the T-cell compartment homologous to human CLL. Leukemia. 2011;25:1452-8.

11. Miller DH, Weber T, Grove R, Wardell C, Horrigan J, Graff O, et al. Firategrast for relapsing remitting multiple sclerosis: a phase 2, randomised, double-blind, placebo-controlled trial. Lancet Neurol. 2012;11:131-9.

12. Heinig K, Gatjen M, Grau M, Stache V, Anagnostopoulos I, Gerlach K, et al. Access to follicular dendritic cells is a pivotal step in murine chronic lymphocytic leukemia B-cell activation and proliferation. Cancer Discov. 2014;4:1448-65.

13. Brachtl G, Sahakyan K, Denk U, Girbl T, Alinger B, Hofbauer SW, et al. Differential bone marrow homing capacity of VLA-4 and CD38 high expressing chronic lymphocytic leukemia cells. PLoS ONE. 2011;6:e23758.

14. Gutjahr JC, Szenes E, Tschech L, Asslaber D, Schlederer M, Roos $\mathrm{S}$, et al. Microenvironment-induced CD44v6 promotes early disease progression in chronic lymphocytic leukemia. Blood. 2018;131:1337-49.

15. Moreno OBT, Zann V, Willson A, Leung P, Connor A. Safety, pharmacokinetics, and pharmacodynamics of ME-401, an oral, potent, and selective inhibitor of phosphatidylinositol 3-kinase P1108, following single ascending dose administration to healthy volunteers. Clin Ther. 2018;40:1855-67. 